\title{
Rapid thermal emittance and quantum efficiency mapping of a cesium telluride cathode in an rf photoinjector using multiple laser beamlets
}

\author{
Lianmin Zheng $\odot,{ }^{1,2}$ Jiahang Shao $\odot,{ }^{2}$ Eric E. Wisniewski $\odot,{ }^{2}$ John G. Power, ${ }^{2}$ Yingchao Du®, ${ }^{1, *}$ \\ Wanming Liu, ${ }^{2}$ Charles E. Whiteford, ${ }^{2}$ Manoel Conde, ${ }^{2}$ Scott Doran, ${ }^{2}$ \\ Chunguang Jing, ${ }^{2,3}$ and Chuanxiang Tang ${ }^{1}$ \\ ${ }^{1}$ Department of Engineering Physics, Tsinghua University Beijing, \\ Beijing 100084, People's Republic of China \\ ${ }^{2}$ High Energy Physics Division, Argonne National Laboratory, Lemont, Illinois 60439, USA \\ ${ }^{3}$ Euclid Techlabs LLC, Bolingbrook, Illinois 60440, USA
}

(Received 6 March 2020; accepted 22 April 2020; published 4 May 2020)

\begin{abstract}
Thermal emittance and quantum efficiency (QE) are key figures of merit of photocathodes, and their uniformity is critical to high-performance photoinjectors. Several QE mapping technologies have been successfully developed; however, there is still a dearth of information on thermal emittance maps. This is because of the extremely time-consuming procedure to gather measurements by scanning a small beam across the cathode with fine steps. To simplify the mapping procedure and to reduce the time required to take measurements, we propose a new method that requires only a single scan of the solenoid current to simultaneously obtain thermal emittance and QE distribution by using a pattern beam with multiple beamlets. In this paper, its feasibility has been confirmed by both beam dynamics simulation and theoretical analysis. The method has been successfully demonstrated in a proof-of-principle experiment using an L-band radio-frequency photoinjector with a cesium telluride cathode. In the experiment, seven beamlets were generated from a microlens array system and their corresponding thermal emittance and QE varied from 0.93 to $1.14 \mu \mathrm{m} / \mathrm{mm}$ and from $4.6 \%$ to $8.7 \%$, respectively. We also discuss the limitations and future improvements of the method in this paper.
\end{abstract}

DOI: 10.1103/PhysRevAccelBeams.23.052801

\section{INTRODUCTION}

Beam brightness, defined by current over emittance, is one of the most important properties of photoinjectors. The continuous improvement of beam brightness over the past few decades has enabled many photoinjector-based machines for scientific research, such as x-ray free electron lasers [1,2], ultrafast electron diffraction and microscopy $[3,4]$, Thomson scattering x-ray sources $[5,6]$, and wakefield acceleration $[7,8]$. The beam brightness of a photoinjector heavily depends on the photocathode, because its thermal emittance (also known as intrinsic emittance, denoted as $\varepsilon_{\text {therm }}$ or $\varepsilon_{\text {therm }, n} \equiv \varepsilon_{\text {therm }} / \sigma_{\text {laser }}$ when normalized by the rms laser spot size) sets the lower boundary of beam emittance and its quantum efficiency (QE) determines the current with a certain incident laser. Recently, intense studies have focused on advanced cathode research and

\footnotetext{
*dych@mail.tsinghua.edu.cn
}

Published by the American Physical Society under the terms of the Creative Commons Attribution 4.0 International license. Further distribution of this work must maintain attribution to the author(s) and the published article's title, journal citation, and DOI. development to obtain low thermal emittance and high QE [9-17].

Most cathode studies work on the average thermal emittance and QE of large areas [18-24]; however, several groups have begun to develop mapping technologies to look into the detailed distributions of these key properties over the cathode [25-34]. Several QE mapping technologies have been developed and applied in photoinjectors, and nonuniform QE distribution has been observed [30-33]. These variations could be caused by localized surface conditions, such as physical and chemical roughness, material defects, and contaminants, etc., $[27,30]$. According to the correlation between QE and thermal emittance [9,35-38], these conditions would also lead to localized variations in thermal emittance.

To the best of our knowledge, however, there is still a lack in thermal emittance mapping technology for photoinjectors. In fact, measuring the thermal emittance of a fixed cathode area using a single beam remains difficult, because all emittance growth factors need to be properly addressed [31,36,39-41]. These factors can be categorized as $\varepsilon_{\text {space }}$, which denotes the emittance growth from space charge [42], and $\varepsilon_{\text {aberration }}$, which denotes the growth caused by aberrations, including dipole, quadrupole, and high-order field 
components inside the radio-frequency (rf) photocathode gun; spherical and chromatic aberrations in the solenoid; and coupled transverse dynamics aberrations [31,41,43-48]. The measurement would be even more challenging and time consuming when the same procedure is repeated in thermal emittance mapping by scanning a single beam across the cathode with fine steps.

In this paper, we propose and experimentally demonstrate a rapid method to map thermal emittance and $\mathrm{QE}$ simultaneously. The basic concept is to use isolated multiple beamlets instead of a single beam in a solenoid scan. With the proper size and separation of the laser beamlets, the generated electron beamlets are distinguishable in a certain current range during the solenoid scan so that their thermal emittance and QE can be measured individually. We believe this method will find broad application in the high-brightness photoinjector community: It can deepen the understanding of the observed nonuniformity, improve simulation accuracy with a realistic distribution of cathode properties, and further increase beam brightness by helping to choose the emission site on the cathode.

This paper is organized as follows. Section II introduces the beam line layout for analysis and experiment. Section III analyzes the method feasibility with beam dynamics simulation and theoretical derivation. Section IV gives more details of the experimental setup. Section V presents the data analysis method and experimental results. Section VI briefly discusses the observed correlation between thermal emittance and QE and studies the current limitations and future improvements of the proposed method. Section VII summarizes the current work.

\section{BEAM LINE LAYOUT}

The front end of the drive beam line at the Argonne Wakefield Accelerator (AWA) facility [49] is used in the beam dynamics analysis and the following experiment, as illustrated in Fig. 1.

The setup includes an L-band 1.6-cell photocathode gun with a cesium telluride cathode, followed by a solenoid



FIG. 1. Beam line setup in the simulation and experiment.
( $2.44 \mathrm{~m}$ from the cathode) and a screen (2.98 $\mathrm{m}$ from the cathode). The incident $248 \mathrm{~nm}$ laser has a Gaussian longitudinal distribution with a full width at half maximum pulse length of $1.5 \mathrm{ps}$. The cathode gradient reaches $62 \mathrm{MV} / \mathrm{m}$ in the routine high-charge operation $[8,50]$. In this study, the cathode gradient is set to $32.5 \mathrm{MV} / \mathrm{m}$ to reduce field emission and to improve the signal-to-noise ratio. The laser injection phase is set to $30^{\circ}$, which is close to the phase of the maximum energy gain under this gradient $\left(37^{\circ}\right)$.

\section{BEAM DYNAMICS SIMULATION AND THEORETICAL ANALYSIS}

This section first summarizes the basic concept of thermal emittance measurement using the solenoid scan technology with a single beam. Next, it describes the beam dynamics simulation and the theoretical analysis we used to study the feasibility of the proposed thermal emittance mapping method with multiple beamlets.

In this section, $e$ and $\beta \gamma m c$ denote the charge and momentum of the electron, respectively; $B_{0}, L, K \equiv$ $\left(e B_{0}\right) /(2 \beta \gamma m c)$, and $K L$ denote the peak magnetic field, effective length, strength, and Larmor angle of the solenoid, respectively.

\section{A. Solenoid scan with a single beam}

In the conventional solenoid scan with a single beam, the beam spot size squared taken on a screen downstream of the solenoid varies with the solenoid strength as [41]

$$
\begin{aligned}
\sigma^{2}= & \left(C-L_{d} K S\right)^{2}\left\langle x_{0}^{2}\right\rangle \\
& +2\left(C-L_{d} K S\right)\left(S / K+C L_{d}\right)\left\langle x_{0} x_{0}^{\prime}\right\rangle \\
& +\left(S / K+C L_{d}\right)^{2}\left\langle x_{0}^{\prime 2}\right\rangle,
\end{aligned}
$$

where $\left\langle x_{0}^{2}\right\rangle,\left\langle x_{0} x_{0}^{\prime}\right\rangle$, and $\left\langle x_{0}^{\prime 2}\right\rangle$ are the beam moments at the solenoid entrance, $C \equiv \cos (K L), S \equiv \sin (K L)$, and $L_{d}$ is the drift length between the solenoid exit and the screen. Therefore, the beam moments can be fitted from the beam size when scanning the solenoid strength, and the normalized emittance at the solenoid entrance can be expressed as

$$
\varepsilon_{n}=\beta \gamma \sqrt{\left\langle x_{0}^{2}\right\rangle\left\langle x_{0}^{\prime 2}\right\rangle-\left\langle x_{0} x_{0}^{\prime}\right\rangle^{2}}
$$

\section{B. Solenoid scan with multiple beamlets}

To reduce the amount of time necessary to collect a measurement by moving a single beam for thermal emittance mapping, it is natural to use a pattern beam with isolated multiple beamlets and obtain the thermal emittance distribution in a single-turn measurement. 


\section{Beam dynamics simulation}

We first conduct the beam dynamics simulation with the ASTRA code [51] to evaluate this concept. The simulation considers three-dimensional field maps with higher-order field components of the photocathode gun and the solenoid $[41,48]$. The space charge is not considered in the simulation, which is valid for thermal emittance measurements with ultralow charge.

For simplicity, we use only two beamlets in the simulation. Each Gaussian-shaped beamlet on the photocathode has a $50 \mu \mathrm{m} \mathrm{rms} \mathrm{spot} \mathrm{size,} \mathrm{and} \mathrm{it} \mathrm{is} \mathrm{cut} \mathrm{at} 150 \mu \mathrm{m}$ radius $(3 \sigma$ cut, denoted as $\left.x_{c, \text { max }}\right)$. The first beamlet is set at the center of the cathode, and the second is offset horizontally by $1.812 \mathrm{~mm}$. The center-to-center distance between the beamlets on the cathode is denoted as $\triangle x_{c}$, as illustrated in Fig. 2(a). The $\varepsilon_{\text {therm, } n}$ of both beamlets is set to $1.05 \mu \mathrm{m} / \mathrm{mm}$ according to a previous experiment [41].

The key challenge in solenoid scans with multiple beamlets is to find a suitable range of the solenoid strength for beam moment fitting in which the beamlets are distinguishable. In this range and at the screen position, we define the center-to-center distance between the electron beamlets as $\triangle x$ and their closest distance as $D_{\min }$, as illustrated in Fig. 2(b). In the ASTRA simulation, as the solenoid strength increases from $B_{0}$ of $0.1859 \mathrm{~T}$, the initially separated beamlets [Fig. 3(a)] begin to merge [Fig. 3(b)], fully overlap [Fig. 3(c)], begin to separate [Fig. 3(d)], and fully separate again when $B_{0}$ is higher than $0.1995 \mathrm{~T}$ [Fig. 3(e)]. When $B_{0}$ is higher than $0.1995 \mathrm{~T}, \Delta x$ [Fig. 4(a)] and $D_{\min }$ [Fig. 4(b)] monotonically increase with the solenoid strength. A positive $D_{\min }$ indicates that the beamlets can be well distinguished. The rms size of each individual beamlet $\sqrt{\left\langle x^{2}\right\rangle}$ reaches a waist at $B_{0}$ of $0.2220 \mathrm{~T}$ [Figs. 3(f) and 4(c)].

With the beam spot sizes in the $B_{0}$ range between 0.2 and $0.25 \mathrm{~T}$, the emittance of the two beamlets is fitted to be only $0.2 \%$ (the first beamlet) and $0.3 \%$ (the second beamlet) higher than the thermal value. This negligible emittance growth is caused by $\varepsilon_{\text {aberration }}$.

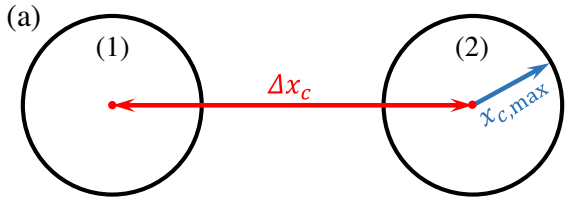

(b)



FIG. 2. Definition of beamlet geometry parameters on the cathode (a) and on the screen after the solenoid (b). (1) and (2) in the figure indicate the two beamlets.
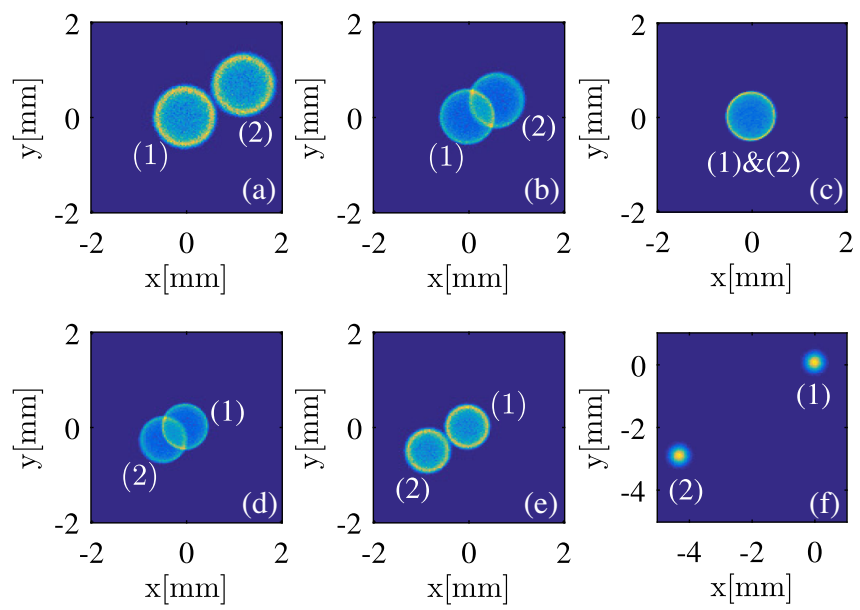

FIG. 3. The simulated beamlet distribution on the screen as a function of $B_{0}$. In (a)-(f), $B_{0}$ is set to $0.1859,0.1900,0.1940$, $0.1970,0.1995$, and $0.2220 \mathrm{~T}$, respectively. (1) and (2) in the figure indicate the two beamlets.
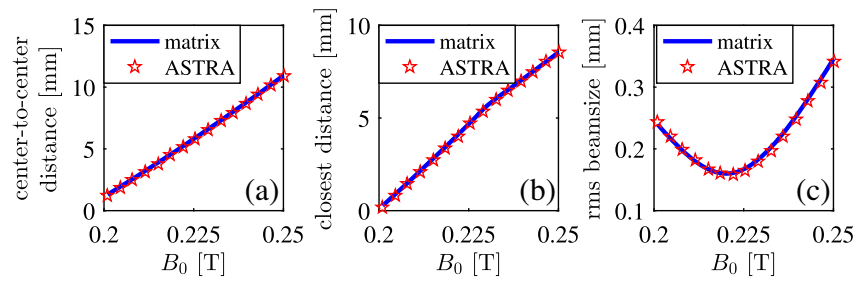

FIG. 4. Beam evolution as a function of $B_{0}$ when $B_{0}$ is higher than 0.1995 T. (a) Center-to-center distance. (b) Closest distance between the two beamlets. (c) The rms beam size. Red stars: ASTRA simulation results. Solid blue lines: Matrix calculation results.

\section{Matrix analysis}

We then employ a start-to-end matrix calculation of the beam dynamics to analyze the solenoid scan with multiple beamlets and give insight into the ASTRA simulation results.

In a transverse Larmor coordinate (i.e., the axis rotates along the solenoid), the motion of an electron can be expressed as [52]

$$
\left[\begin{array}{c}
x \\
p_{x}
\end{array}\right]=\left[\begin{array}{ll}
R_{11} & R_{12} \\
R_{21} & R_{22}
\end{array}\right]\left[\begin{array}{c}
x_{c} \\
p_{x_{c}}
\end{array}\right],
$$

where $x_{c}$ and $x$ are the beam position on the cathode and the screen, respectively; $p_{x_{c}}=\beta_{x_{c}} \gamma$ and $p_{x}=\beta_{x} \gamma$ are the corresponding normalized transverse momenta; and $R_{i j}$ is the transfer matrix element from the cathode to the screen.

Based on Eq. (3), the final position $x$ is written as

$$
x=R_{11} x_{c}+R_{12} p_{x_{c}} .
$$

For isotropic emission on the cathode with $\bar{p}_{x_{c}}=0$ and $\left\langle x_{c} p_{x_{c}}\right\rangle=0$, the beam position $\bar{x}$ and the rms beam size square $\left\langle x^{2}\right\rangle$ on the screen can be expressed as 

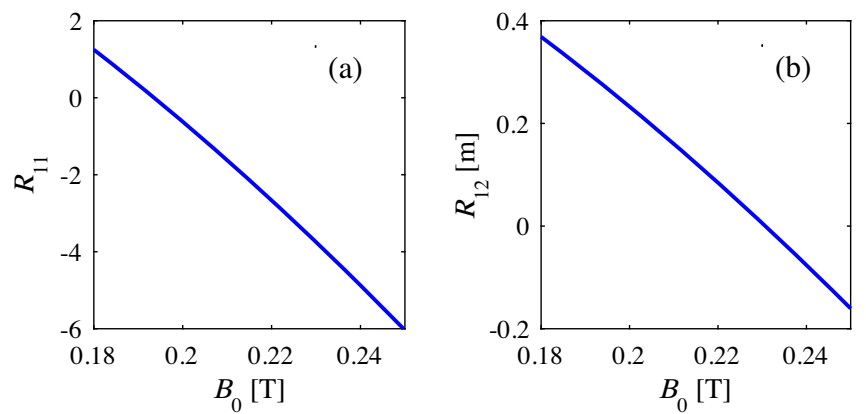

FIG. 5. (a) $R_{11}$ and (b) $R_{12}$ as a function of $B_{0}$.

$$
\begin{aligned}
\bar{x} & =R_{11} \bar{x}_{c}, \\
\left\langle x^{2}\right\rangle & =R_{11}^{2}\left\langle x_{c}^{2}\right\rangle+R_{12}^{2}\left\langle p_{x_{c}}^{2}\right\rangle .
\end{aligned}
$$

Therefore, the closest distance between the two beamlets can be expressed as

$$
D_{\min }=\left|R_{11} \triangle x_{c}\right|-2\left|R_{11} x_{c, \max }\right|-2\left|R_{12} p_{x_{c, \max }}\right|,
$$

where $p_{x_{c, \max }}$ is the maximum normalized transverse momentum on the cathode.

We follow the method introduced in Ref. [53] to calculate the transfer matrix from the cathode to the screen. In this method, the superimposed one-dimensional $\mathrm{rf}$ and solenoid fields are considered, and the transfer matrix is computed without solving eigenfunction expansions or numerical derivatives. In the calculation, we apply the same beam line parameters as in the ASTRA simulation, except for the three-dimensional field maps. The use of a one-dimensional field is valid, because $\varepsilon_{\text {aberration }}$ is negligible based on the previous simulation. The resultant $R_{11}$ and $R_{12}$ are illustrated in Fig. 5.

The beam parameters are also set to be the same as the ones used in the ASTRA simulation: $\sqrt{\left\langle x_{c}^{2}\right\rangle}$ is $50 \mu \mathrm{m}, x_{c, \max }$ is $150 \mu \mathrm{m}, \sqrt{\left\langle p_{x_{c}}^{2}\right\rangle}$ is equal to $\varepsilon_{\text {therm, } n}$ of $1.05 \mu \mathrm{m} / \mathrm{mm}$, $p_{x_{c, \max }}$ is $1.82 \times 10^{-3}\left(\sqrt{3\left\langle p_{x_{c}}^{2}\right\rangle}\right.$ for isotropic emission $)$, and $\bar{x}_{c}=0$ and $1.812 \mathrm{~mm}$ for the two beamlets $\left(\triangle x_{c}=\right.$ $1.812 \mathrm{~mm})$, respectively.

The calculation has been compared with the ASTRA simulation. The two beamlets fully overlap when $R_{11}=0$. The corresponding $B_{0}$ was found to be $0.194 \mathrm{~T}$ based on Fig. 5(a), which agrees well with the simulation result in Fig. 3(c). When the two beamlets are distinguishable $\left(D_{\min }>0\right)$, their center-to-center distance, closest distance, and rms beam size are calculated according to Eqs. (5) and (6). These equations show good agreement with the ASTRA simulation, as illustrated in Fig. 4.

\section{EXPERIMENTAL SETUP}

Figure 1 illustrates the experimental setup at AWA. At the photocathode gun exit, the electron beams reached $3.3 \mathrm{MeV}$ and were focused by the solenoid onto a retractable yttriumaluminum-garnet (YAG) screen perpendicular to the beam

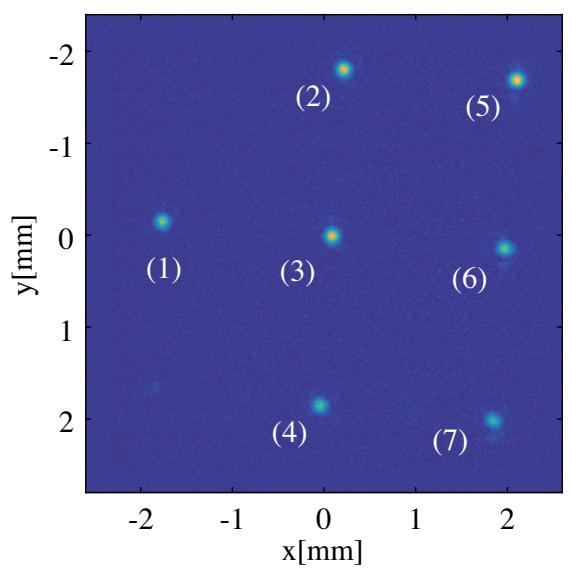

FIG. 6. Laser transverse pattern observed on the virtual cathode. The brightness variation among the laser beamlets was caused by the incident laser, as well as nonideal conditions of the MLA system. The third beamlet was centered on the cathode.

line. The beam images were reflected by a $45^{\circ}$ mirror after the YAG screen and captured by a PI-MAX Intensified CCD camera (ICCD) [54]. The shutter width of the camera was set to $100 \mathrm{~ns}$ to improve the signal-to-noise ratio. The spatial resolution of the camera was $\sim 60 \mu \mathrm{m}$, measured with a standard USAF target. A calibrated strip line beam position monitor (BPM) downstream was used to measure the charge with a sensitivity of $\sim 40 \mathrm{mV}(1 \mathrm{pC})[41,48]$. The minimum detectable charge of the multiple beamlets is therefore $0.05 \mathrm{pC}(2 \mathrm{mV})$.

The multiple laser beamlets were generated by a microlensarray (MLA) system [55,56]. After passing through a pair of MLAs and three convex lenses, the incident ultraviolet (UV) laser was redistributed to yield a pattern with twodimensional arrays of beamlets at the iris location. The iris was set to select a few beamlets from the entire pattern. Then the pattern was imaged onto the cathode by a pair of convex and concave lenses. The UV laser energy loss of the MLAs and the lenses was $~ 90 \%$. On the virtual cathode (not shown in Fig. 1), the laser pattern was captured by a UV camera with a high spatial resolution of $7.5 \mu \mathrm{m}$ per pixel. In this proof-of-principle experiment, the system successfully produced a pattern with seven laser beamlets, as illustrated in Fig. 6. Each beamlet had a Gaussian-like transverse distribution with an $\mathrm{rms}$ spot size of $\sim 50 \mu \mathrm{m}$. The distance between two adjacent beamlets was $1.812 \mathrm{~mm}$. The laser energy of the entire pattern was measured by a UV power meter. The energy of each laser beamlet was calculated according to the beamlet's relative brightness on the UV camera.

\section{EXPERIMENTAL RESULTS}

\section{A. Thermal emittance mapping}

The beam images on the screen under different solenoid settings are shown in Fig. 7. The seven beamlets fully 



FIG. 7. Beam images as a function of solenoid strength. $B_{0}$ in (a)-(d) is $0.2045,0.2170,0.2294$, and $0.2530 \mathrm{~T}$, respectively. The electron beamlets are marked in (c) following the same laser beamlet index as in Fig. 6.

overlap when $B_{0}=0.1940 \mathrm{~T}$, become distinguishable when $B_{0}>0.2045 \mathrm{~T}$, and reach the waist when $B_{0}=$ $0.2294 \mathrm{~T}$. These solenoid strengths show reasonable agreement with the ASTRA simulation and the theoretical analysis results.

We calculated the beam size of each electron beamlet using the following procedure. (i) Background field emission images without laser illumination were subtracted to improve the signal-to-noise ratio. (ii) Each beamlet was manually selected, and its projected distributions in the $x$ and $y$ directions were Gaussian fitted to obtain the coarse rms beam sizes, denoted $\sigma_{g x}$ and $\sigma_{g y}$, respectively. (iii) For each beamlet, the image within $3 \sqrt{\sigma_{g x} \sigma_{g y}}$ of its center was preserved, while the rest was set to zero. (iv) The cut image was projected in the $x$ and $y$ directions again, and the accurate rms beam sizes were calculated by selecting the central $95 \%$ of the entire area under the projection curve (i.e., $5 \%$ charge cut of the most outward part of the beamlet) [57]. (v) The rms beam size was calculated as the geometric average of the sizes in the $x$ and $y$ directions: $\sigma=\sqrt{\sigma_{x} \sigma_{y}}$.

Six images were taken under each solenoid strength, and we calculated the emittance using the following procedure. (i) For each $B_{0}$, the rms beam size fluctuated due to machine jitter, and its distribution was assumed to be Gaussian. The average value and the standard deviation of the distribution were calculated from the six images as $\bar{\sigma}$ and $\delta \sigma$, respectively. (ii) The average emittance of each beamlet was fitted using $\bar{\sigma}$ and $B_{0}$ according to Eq. (1). (iii) Another set of beam sizes was generated from the Gaussian distributions and applied to Eq. (1) for emittance fitting. (iv) Step (iii) was repeated multiple times. The fitted emittance was weighted according to the probability of each set of beam sizes in the Gaussian distributions, from which the standard deviation of emittance can be calculated. It should be noted that the error bar in fitting is negligible when compared to the standard
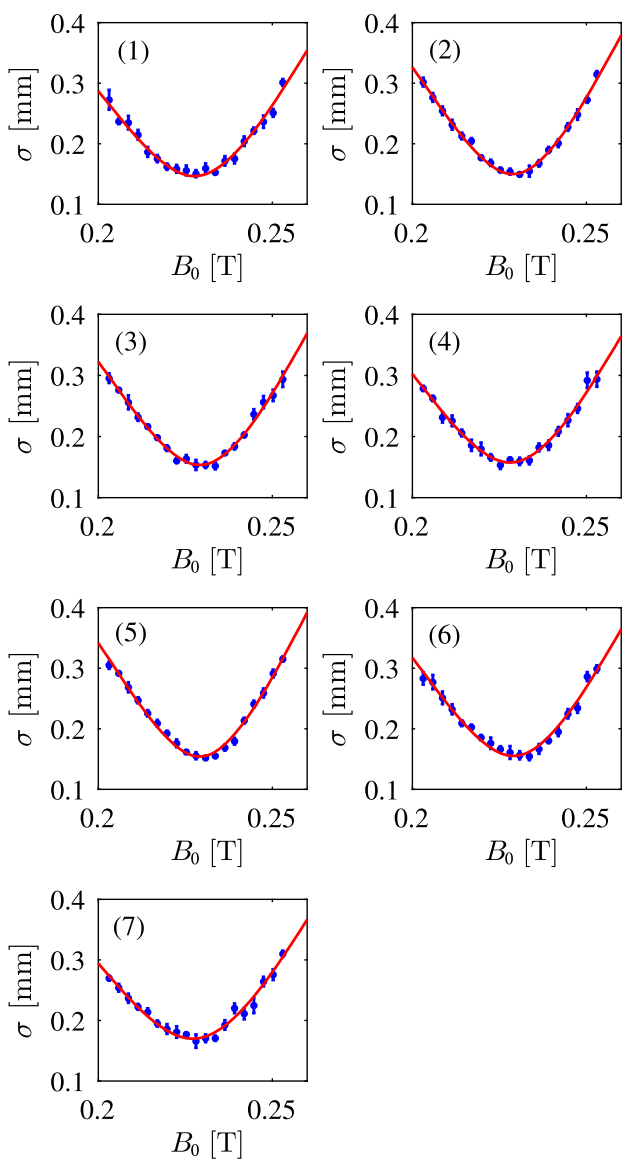

FIG. 8. The rms beam sizes of the seven beamlets (marked by number) as a function of $B_{0}$. Blue dots: Experimental data. The error bar at each $B_{0}$ denotes the standard deviation of the measured beam size. Red lines: Fitting with $\bar{\sigma}$ according to Eq. (1).

deviation caused by machine jitter in our measurement. The rms beam sizes of the seven beamlets as a function of $B_{0}$ are plotted in Fig. 8.

The influence of $\varepsilon_{\text {aberration }}$ on the thermal emittance measurement has been studied in ASTRA using the same settings as in Sec. III. B. 1. The simulation results indicate the influence is negligible: The emittance growth is $0.2 \%$ for beamlet 3 (at the cathode center); $0.3 \%$ for beamlets 1 , 2 , 4, and 6 (1.812 $\mathrm{mm}$ off axis); and $0.4 \%$ for beamlets 5 and 7 (2.563 $\mathrm{mm}$ off axis).

The influence of $\varepsilon_{\text {space }}$ was experimentally minimized by reducing the total charge of the pattern beam. The total incident laser energy was controlled by neutral density filters (NDFs) before the MLA system. The charge of each beamlet can be calculated from the total charge measured by the calibrated BPM and the relative brightness of the beamlet over the entire pattern on the YAG screen. For example, the measured emittance of beamlet 1 as a function of its charge is shown in Fig. 9. The measured emittance converged when the beamlet charge was lower than $0.02 \mathrm{pC}$, which indicates that $\varepsilon_{\text {space }}$ is negligible and $\varepsilon_{n} \approx$ $\varepsilon_{\text {therm }}$ below this charge level. 




FIG. 9. Measured emittance of beamlet 1 as a function of the beamlet charge. Black circles with error bars: Experimental data. Dashed green line: Converged emittance.

The measured thermal emittance of the seven beamlets is summarized in Table I. In this table, the spot size of each laser beamlet is calculated following the same steps [steps (ii)-(v)] as the electron beamlets. Its average value and standard deviation are calculated using 20 images taken by the UV camera. The resultant thermal emittance varies from 0.934 to $1.142 \mu \mathrm{m} / \mathrm{mm}$. Their average value is close to the previous experimental result of $1.05 \mu \mathrm{m} / \mathrm{mm}$ using a large single beam with $3 \mathrm{~mm}$ diameter [41]. We notice that these values are higher than some of the previously reported results of thermal emittance of cesium telluride cathodes [21-23]. This may be caused by various factors, such as cathode preparation procedures, surface conditions, and operation conditions.

\section{B. QE mapping}

The QE mapping is straightforward with isolated multiple beamlets, because the laser energy and the charge of each beamlet can be derived based on experimental measurement. For example, Fig. 10 shows the charge of beamlet 1 when gradually reducing the laser energy with NDFs. The linearity between the charge and the laser energy confirms single-photon emission, and its slope is

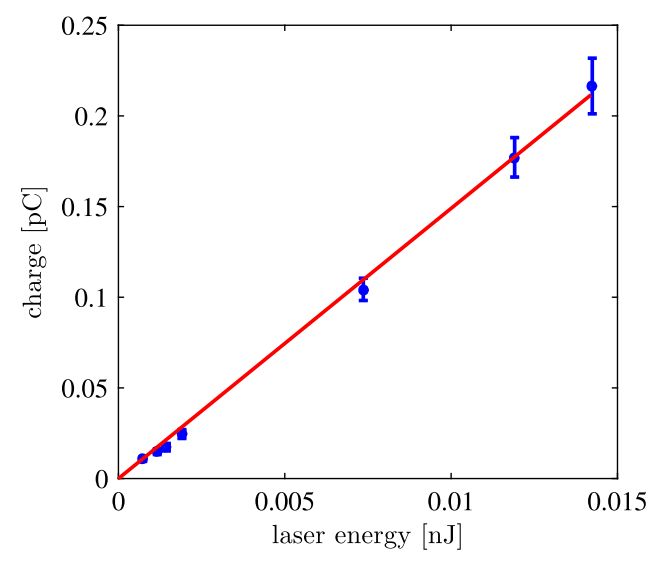

FIG. 10. Measured charge as a function of the laser energy for beamlet 1. Blue dots: Experimental data. The error bar denotes the standard deviation of the measured charge. Red line: Linear fitting.

used to calculate the $\mathrm{QE}$. The $\mathrm{QE}$ of the seven beamlets is also summarized in Table I.

\section{DISCUSSION}

\section{A. Dependence of thermal emittance on QE}

Based on Table I, we can see that beamlets with higher QE usually have higher thermal emittance. This trend is illustrated in Fig. 11.

In a metallic photocathode, the thermal emittance and QE depend on electron excess energy $h \nu-\phi_{\text {eff. }}$ This can be described by Dowell's three-step model [35] as follows:

$$
\begin{aligned}
\varepsilon_{\text {therm }, n} & \propto \sqrt{h \nu-\phi_{\mathrm{eff}}}, \\
\mathrm{QE} & \propto\left(h \nu-\phi_{\mathrm{eff}}\right)^{2},
\end{aligned}
$$

where $h \nu$ is the incident photon energy and $\phi_{\text {eff }}$ is the effective work function.

In a semiconductor photocathode, the first equation holds valid by assuming that most photoelectrons emit from the valence band $[9,36]$. However, the second one is still controversial due to the complicated emission mechanism [37,38]. Reference [38] suggested a modified version:

TABLE I. Experimental results of the rms spot size of the laser beamlet $\sigma_{\text {laser }}$, thermal emittance $\varepsilon_{\text {therm }}, \varepsilon_{\text {therm, }, n}$, and QE of the seven beamlets. The error bar denotes the standard deviation of the measurement.

\begin{tabular}{lcccc}
\hline \hline Beamlet no. & $\sigma_{\text {laser }}(\mu \mathrm{m})$ & $\varepsilon_{\text {therm }}(\mu \mathrm{m})$ & $\varepsilon_{\text {therm }, n}(\mu \mathrm{m} / \mathrm{mm})$ & QE $(\%)$ \\
\hline 1 & $48.4 \pm 0.5$ & $0.0452 \pm 0.0038$ & $0.934 \pm 0.079$ & $4.59 \pm 0.66$ \\
2 & $49.8 \pm 0.4$ & $0.0518 \pm 0.0031$ & $1.040 \pm 0.063$ & $7.17 \pm 0.80$ \\
3 & $50.2 \pm 0.4$ & $0.0515 \pm 0.0034$ & $1.026 \pm 0.068$ & $6.15 \pm 0.57$ \\
4 & $49.7 \pm 0.7$ & $0.0500 \pm 0.0041$ & $1.006 \pm 0.084$ & \\
5 & $48.7 \pm 0.5$ & $0.0556 \pm 0.0026$ & $1.142 \pm 0.055$ & $8.60 \pm 0.79$ \\
6 & $49.9 \pm 0.5$ & $0.0510 \pm 0.0042$ & $1.022 \pm 0.085$ & $5.63 \pm 0.79$ \\
7 & $51.8 \pm 0.9$ & $0.0520 \pm 0.0033$ & $1.004 \pm 0.066$ & $5.47 \pm 0.55$ \\
\hline \hline
\end{tabular}




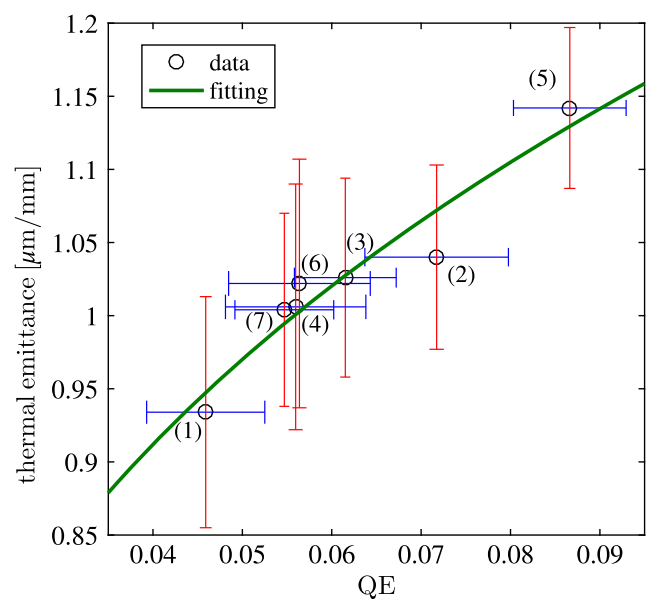

FIG. 11. Dependence of thermal emittance on QE. Black circles with error bars: Experiment data of the seven beamlets (marked by number). Green line: Least-square fitting.

$$
\mathrm{QE} \propto\left(h \nu-\phi_{\mathrm{eff}}\right)^{p}
$$

where $p$ is a constant.

From the experimental data, the dependence of thermal emittance on $\mathrm{QE}$ was least-square fitted as $\varepsilon_{\text {therm, } n}=$ $2.223 \times \sqrt[3.61]{\mathrm{QE}}$, which resulted in $p=1.805$. This number is within the large range of $p$ from 1.3 to 4.6 extracted from previous studies $[14,58,59]$. The large variation of $p$ may be caused by preparation procedures, surface conditions, operation conditions, and other factors. The physical understanding of the constant $p$ requires more fundamental research in the future.

\section{B. Mapping limitation and future improvement}

We measured the thermal emittance and QE of seven isolated spots near the cathode center in this proof-ofprinciple experiment. In future studies, the mapping method could be improved with a larger area, higher resolution, and higher density.

The mapping area is defined as the maximum boundary that the beamlets can cover on the cathode. When the space charge effect is negligible, the mapping area will be limited by the emittance growth from the aberration terms. We use the ASTRA simulation with a single off-axis beamlet to evaluate the growth. In the simulation, the beam line parameters and the size or emittance of the beamlet are kept the same as the ones in Sec. III. B. 1. A quad corrector, containing a pair of normal and skew quadrupoles, has been added to correct the coupled transverse dynamics aberration. More details of the quad corrector can be found in Refs. [41,48]. The simulation results show monotonic emittance growth when increasing the beam offset, as illustrated in Fig. 12. The emittance growth reaches $10 \%$ when the beam offset is $12.5 \mathrm{~mm}$.

The mapping resolution is defined as the smallest area that can be measured by a beamlet. It can be improved by

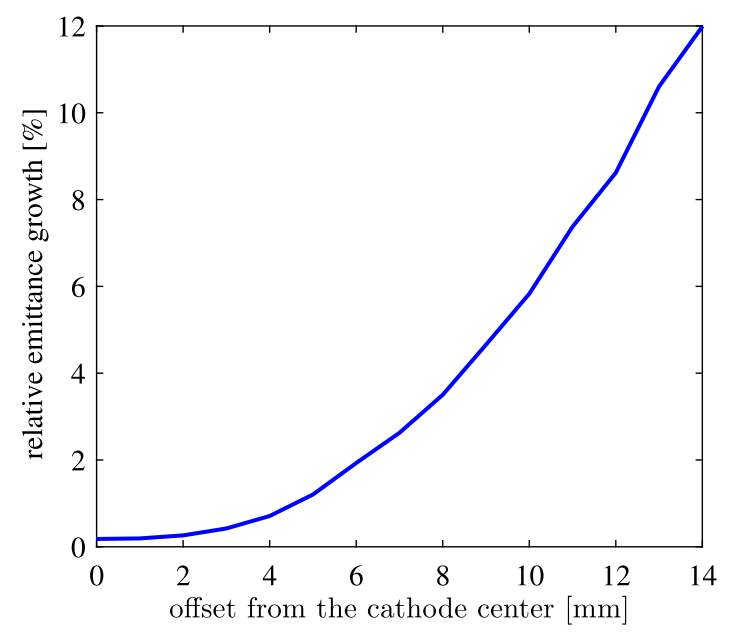

FIG. 12. $\left(\varepsilon_{n}-\varepsilon_{\text {therm }}\right) / \varepsilon_{\text {therm }}$ as a function of the beamlet offset on the cathode.

using smaller laser spots, in general, but will be eventually limited by the system resolution in a given experiment, such as the UV camera that determines the laser beamlet spot size and the YAG screen that determines the electron beamlet spot size.

The mapping density is defined as the number of sampling beamlets per unit area. Unlike some QE mapping methods, in which a single beam scans across the cathode continuously, the initial beamlets in the proposed method need to be separated by a certain distance when placed on the cathode. The minimum separation, or the highest density, is physically limited by the beamlet overlapping issue.

Based on Eq. (6), the dependence of the closest distance between two adjacent electron beamlets $D_{\min }$ on the laser beamlet separation $\triangle x_{c}$ and the solenoid strength $B_{0}$ can be calculated, as illustrated in Fig. 13(a). The beam line and beam parameters, other than the initial separation, remain the same as those in Sec. III. B. 1. The minimum initial distance in the calculation is set to $300 \mu \mathrm{m}$, which is twice the beamlet radius. When the initial separation is larger, there will always be a $B_{0}$ range in which the beamlets are distinguishable $\left(D_{\min }>0\right)$. In principle, the beam moments can be fitted out in this range and the emittance can be calculated accordingly. In practice, however, it is desirable to include both sides around the beam waist in fitting, in order to obtain accurate results [60], as illustrated in Figs. 4(c) and 8. In Fig. 13(a), when $\triangle x_{c}$ is larger than $415 \mu \mathrm{m}$, both sides around the beam waist have a distinguishable range.

Even when both sides are available, there are still several different criteria to select the $B_{0}$ range to improve the fitting accuracy [61-63]. For example, Ref. [62] selects the range in such a way that the maximum beam sizes at both sides are twice that of the waist. From Fig. 13(b), we learn that the maximum beam size for certain $\triangle x_{c}$ is limited by the low $B_{0}$ end. In general, larger $\triangle x_{c}$ leads to a larger beam size at this end, as illustrated in Fig. 14. Further discussion 

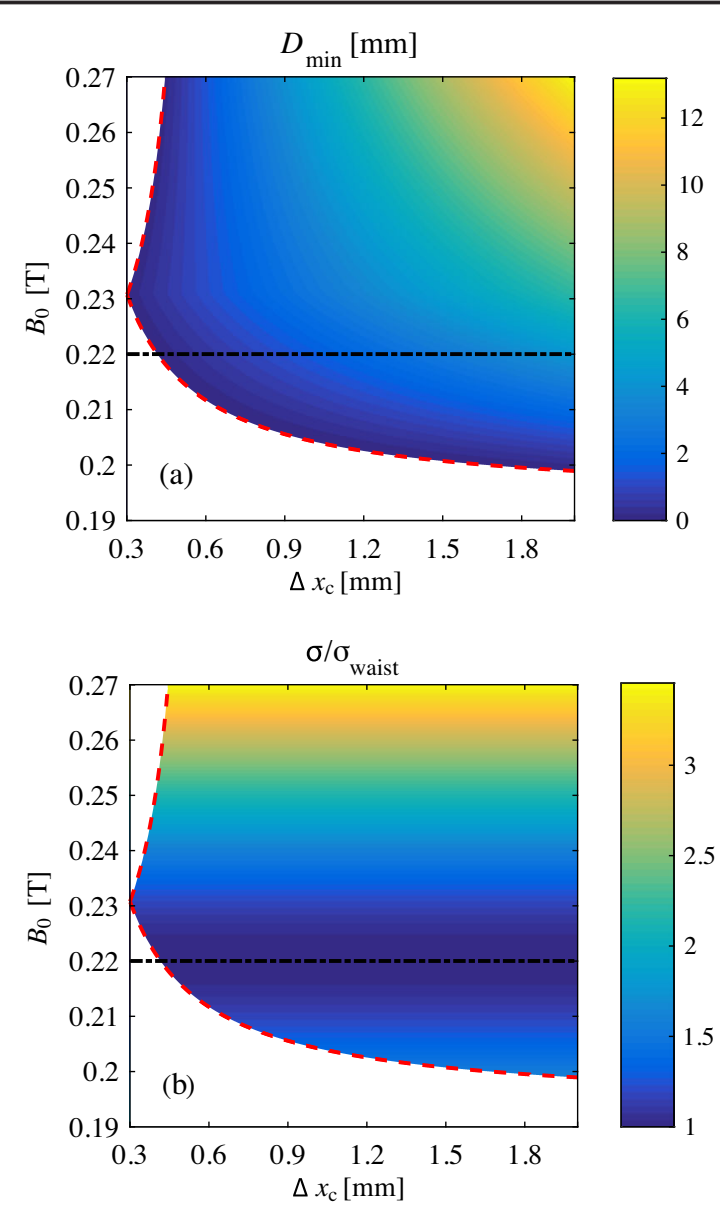

FIG. 13. Dependence of (a) $D_{\text {min }}$ and (b) $\sigma / \sigma_{\text {waist }}$ on $\Delta x_{c}$ and $B_{0}$. The white areas indicate the beamlets cannot be distinguished. Black dotted line: Solenoid strength when the beam size reaches a waist.

of the range selecting criteria is beyond the scope of the current study. It should be noted that, in addition to the selecting criteria, the minimum separation also depends on the initial laser spot size and the thermal emittance.



FIG. 14. Maximum $\sigma / \sigma_{\text {waist }}$ at the low $B_{0}$ end of the distinguishable range as a function of $\triangle x_{c}$.

\section{SUMMARY}

In summary, we introduce a rapid thermal emittance and QE mapping method that uses multiple beamlets in a solenoid scan. Its feasibility is supported by beam dynamics simulations and theoretical analysis. In a proof-of-principle experiment using an L-band rf photoinjector with a cesium telluride cathode, seven beamlets with $50 \mu \mathrm{m}$ rms beam size and $1.812 \mathrm{~mm}$ separation were generated and their thermal emittance (varying from 0.93 to $1.14 \mu \mathrm{m} / \mathrm{mm}$ ) and QE (varying from $4.6 \%$ to $8.7 \%$ ) were successfully measured. The range, resolution, and the density of the proposed method can be improved by using smaller laser beamlets with denser separation in a larger area, which could be achieved by optimizing the MLA system. The ultimate performance will be limited by the emittance growth from the aberration terms, the system resolution, and the beamlet overlapping issue.

\section{ACKNOWLEDGMENTS}

The work at AWA is funded through the U.S. Department of Energy (DOE) Office of Science under Contract No. DE-AC02-06CH11357. The work is also supported by the National Natural Science Foundation of China (NSFC) No. 11435015 and No. 11375097.

[1] P. Emma, R. Akre, J. Arthur, R. Bionta, C. Bostedt, J. Bozek, A. Brachmann, P. Bucksbaum, R. Coffee, F.-J. Decker et al., First lasing and operation of an ångstromwavelength free-electron laser, Nat. Photonics 4, 641 (2010).

[2] W. Ackermann, G. Asova, V. Ayvazyan, A. Azima, N. Baboi, J. Bähr, V. Balandin, B. Beutner, A. Brandt, A. Bolzmann et al., Operation of a free-electron laser from the extreme ultraviolet to the water window, Nat. Photonics 1, 336 (2007).

[3] S. P. Weathersby, G. Brown, M. Centurion, T. F. Chase, R. Coffee, J. Corbett, J. P. Eichner, J. C. Frisch, A. R. Fry, M. Gühr et al., Mega-electron-volt ultrafast electron diffraction at SLAC national accelerator laboratory, Rev. Sci. Instrum. 86, 073702 (2015).

[4] R. Li, C. Tang, Y. Du, W. Huang, Q. Du, J. Shi, L. Yan, and X. Wang, Experimental demonstration of high quality mev ultrafast electron diffraction, Rev. Sci. Instrum. 80, 083303 (2009).

[5] Y. Du, L. Yan, J. Hua, Q. Du, Z. Zhang, R. Li, H. Qian, W. Huang, H. Chen, and C. Tang, Generation of first hard $\mathrm{X}$-ray pulse at Tsinghua Thomson scattering X-ray source, Rev. Sci. Instrum. 84, 053301 (2013).

[6] D. J. Gibson, F. Albert, S. G. Anderson, S. M. Betts, M. J. Messerly, H. H. Phan, V. A. Semenov, M. Y. Shverdin, A. M. Tremaine, F. V. Hartemann et al., Design and operation of a tunable MeV-level Compton-scattering-based $\gamma$-ray source, Phys. Rev. Accel. Beams 13, 070703 (2010).

[7] R. J. England, R. J. Noble, K. Bane, D. H. Dowell, C.-K. Ng, J. E. Spencer, S. Tantawi, Z. Wu, R. L. Byer, E. Peralta 
et al., Dielectric laser accelerators, Rev. Mod. Phys. 86, 1337 (2014).

[8] X. Lu, M. A. Shapiro, I. Mastovsky, R. J. Temkin, M. Conde, J. G. Power, J. Shao, E. E. Wisniewski, and C. Jing, Generation of High-Power, Reversed-Cherenkov Wakefield Radiation in a Metamaterial Structure, Phys. Rev. Lett. 122, 014801 (2019).

[9] D. Dowell, I. Bazarov, B. Dunham, K. Harkay, C. Hernandez-Garcia, R. Legg, H. Padmore, T. Rao, J. Smedley, and W. Wan, Cathode R\&D for future light sources, Nucl. Instrum. Methods Phys. Res., Sect. A 622, 685 (2010).

[10] L. Cultrera, S. Karkare, H. Lee, X. Liu, I. Bazarov, and B. Dunham, Cold electron beams from cryocooled, alkali antimonide photocathodes, Phys. Rev. Accel. Beams 18, 113401 (2015).

[11] J. Feng, S. Karkare, J. Nasiatka, S. Schubert, J. Smedley, and H. Padmore, Near atomically smooth alkali antimonide photocathode thin films, J. Appl. Phys. 121, 044904 (2017).

[12] L. Cultrera, C. Gulliford, A. Bartnik, H. Lee, and I. Bazarov, Ultra low emittance electron beams from multi-alkali antimonide photocathode operated with infrared light, Appl. Phys. Lett. 108, 134105 (2016).

[13] H. Lee, L. Cultrera, and I. Bazarov, Intrinsic emittance reduction in transmission mode photocathodes, Appl. Phys. Lett. 108, 124105 (2016).

[14] M. Gaowei, J. Sinsheimer, D. Strom, J. Xie, J. Cen, J. Walsh, E. Muller, and J. Smedley, Codeposition of ultrasmooth and high quantum efficiency cesium telluride photocathodes, Phys. Rev. Accel. Beams 22, 073401 (2019).

[15] J. Maxson, L. Cultrera, C. Gulliford, and I. Bazarov, Measurement of the tradeoff between intrinsic emittance and quantum efficiency from a NaKSb photocathode near threshold, Appl. Phys. Lett. 106, 234102 (2015).

[16] G. Wang, P. Yang, N. A. Moody, and E. R. Batista, Overcoming the quantum efficiency-lifetime tradeoff of photocathodes by coating with atomically thin two-dimensional nanomaterials, npj 2D Mater. Appl. 2, 17 (2018).

[17] P. Musumeci, J. G. Navarro, J. B. Rosenzweig, L. Cultrera, I. Bazarov, J. Maxson, S. Karkare, and H. Padmore, Advances in bright electron sources, Nucl. Instrum. Methods Phys. Res., Sect. A 907, 209 (2018).

[18] H. J. Qian, C. Li, Y. C. Du, L. X. Yan, J. F. Hua, W. H. Huang, and C.X. Tang, Experimental investigation of thermal emittance components of copper photocathode, Phys. Rev. Accel. Beams 15, 040102 (2012).

[19] H. Lee, S. Karkare, L. Cultrera, A. Kim, and I. V. Bazarov, Review and demonstration of ultra-low-emittance photocathode measurements, Rev. Sci. Instrum. 86, 073309 (2015).

[20] M. C. Divall, E. Prat, S. Bettoni, C. Vicario, A. Trisorio, T. Schietinger, and C. P. Hauri, Intrinsic emittance reduction of copper cathodes by laser wavelength tuning in an $\mathrm{rf}$ photoinjector, Phys. Rev. Accel. Beams 18, 033401 (2015).

[21] D. Filippetto, H. Qian, and F. Sannibale, Cesium telluride cathodes for the next generation of high-average current high-brightness photoinjectors, Appl. Phys. Lett. 107, 042104 (2015).
[22] E. Prat, S. Bettoni, H.-H. Braun, R. Ganter, and T. Schietinger, Measurements of copper and cesium telluride cathodes in a radio-frequency photoinjector, Phys. Rev. Accel. Beams 18, 043401 (2015).

[23] D. Sertore, D. Favia, P. Michelato, L. Monaco, and P. Pierini, Cesium telluride and metals photoelectron thermal emittance measurements using a time-of-flight spectrometer, in Proceedings of the 9th European Particle Accelerator Conference, Lucerne, 2004 (JACoW, Geneva, Switzerland, 2004), pp. 408-410.

[24] S. Lederer, G. Asova, J. W. Baehr, K. Boyanov, C. Boulware, H. J. Grabosch, M. Haenel, Y. Ivanisenko, S. Khodyachykh, $\mathrm{S}$. Korepanov et al., Investigations on the thermal emittance of Cs2Te photocathodes at PITZ, in Proceedings of the 29th Free Electron Laser Conference, Novosibirsk, Russia (JACoW, Geneva, Switzerland, 2007), pp. 350-353.

[25] K. L. Jensen, J. J. Petillo, E. J. Montgomery, Z. Pan, D. W. Feldman, P. G. O'Shea, N. A. Moody, M. Cahay, J. E. Yater, and J. L. Shaw, Application of a general electron emission equation to surface nonuniformity and current density variation, J. Vac. Sci. Technol. B 26, 831 (2008).

[26] A.E. Blaugrund, Measurements of electron emission uniformity from cathodes in high-voltage electron beam diodes, Appl. Phys. Lett. 83, 1264 (2003).

[27] G. Gevorkyan, S. Karkare, S. Emamian, I. V. Bazarov, and H. A. Padmore, Effects of physical and chemical surface roughness on the brightness of electron beams from photocathodes, Phys. Rev. Accel. Beams 21, 093401 (2018).

[28] S. Karkare and I. Bazarov, Effects of surface nonuniformities on the mean transverse energy from photocathodes, Phys. Rev. Applied 4, 024015 (2015).

[29] K. L. Jensen, D. A. Shiffler, J. J. Petillo, Z. Pan, and J. W. Luginsland, Emittance, surface structure, and electron emission, Phys. Rev. Accel. Beams 17, 043402 (2014).

[30] B. C. Riddick, E. J. Montgomery, R. B. Fiorito, H. D. Zhang, A. G. Shkvarunets, Z. Pan, and S. A. Khan, Photocathode quantum efficiency mapping at high resolution using a digital micromirror device, Phys. Rev. Accel. Beams 16, 062802 (2013).

[31] L. Zheng, Y. Du, Z. Zhang, H. Qian, L. Yan, J. Shi, Z. Zhang, Z. Zhou, X. Wu, X. Su et al., Development of s-band photocathode rf guns at Tsinghua University, Nucl. Instrum. Methods Phys. Res., Sect. A 834, 98 (2016).

[32] D. Wu, D. Xiao, J. Wang, Q. Pan, X. Luo, K. Zhou, C. Lao, X. Li, S. Lin, P. Li, H. Zhang, L. Yan, H. Wang, X. Yang, M. L. C. Tang, D. Wang, Q. Wang, Z. Qi, and J. Zhang, In situ high resolution real-time quantum efficiency imaging for photocathodes, arXiv:1710.08148.

[33] K. Kabra, S. Li, F. Cropp, T. J. Lane, P. Musumeci, and D. Ratner, Mapping photocathode quantum efficiency with ghost imaging, Phys. Rev. Accel. Beams 23, 022803 (2020).

[34] P.-W. Huang, H. Qian, Y. Chen, D. Filippetto, M. Gross, I. Isaev, C. Koschitzki, M. Krasilnikov, S. Lal, X. Li et al., Single shot cathode transverse momentum imaging in high brightness photoinjectors, arXiv:2002.06837.

[35] D. H. Dowell and J. F. Schmerge, Quantum efficiency and thermal emittance of metal photocathodes, Phys. Rev. Accel. Beams 12, 074201 (2009). 
[36] E. Prat, S. Bettoni, H.-H. Braun, M. C. Divall, and T. Schietinger, Measurements of intrinsic emittance dependence on rf field for copper photocathodes, Phys. Rev. Accel. Beams 18, 063401 (2015).

[37] H. Xie, I. Ben-Zvi, T. Rao, T. Xin, and E. Wang, Experimental measurements and theoretical model of the cryogenic performance of bialkali photocathode and characterization with Monte Carlo simulation, Phys. Rev. Accel. Beams 19, 103401 (2016).

[38] N. A. Moody, K. L. Jensen, A. Shabaev, S. G. Lambrakos, J. Smedley, D. Finkenstadt, J. M. Pietryga, P. M. Anisimov, V. Pavlenko, E. R. Batista et al., Perspectives on designer photocathodes for x-ray free-electron lasers: Influencing emission properties with heterostructures and nanoengineered electronic states, Phys. Rev. Applied 10, 047002 (2018).

[39] V. Miltchev, J. Baehr, H. J. Grabosch, J. H. Han, M. Krasilnikov, and A. Oppelt, Measurements of thermal emittance for cesium telluride photocathodes at Pitz, in Proceedings of the Free Electron Laser Conference (FEL'05), Stanford, California (JACoW, Geneva, Switzerland, 2005).

[40] C. P. Hauri, R. Ganter, F. Le Pimpec, A. Trisorio, C. Ruchert, and H. H. Braun, Intrinsic Emittance Reduction of an Electron Beam from Metal Photocathodes, Phys. Rev. Lett. 104, 234802 (2010).

[41] L. Zheng, J. Shao, Y. Du, J. G. Power, E. E. Wisniewski, W. Liu, C. E. Whiteford, M. Conde, S. Doran, C. Jing et al., Overestimation of thermal emittance in solenoid scans due to coupled transverse motion, Phys. Rev. Accel. Beams 21, 122803 (2018).

[42] C. Limborg-Deprey and P. R. Bolton, Optimum electron distributions for space charge dominated beams in photoinjectors, Nucl. Instrum. Methods Phys. Res., Sect. A 557, 106 (2006).

[43] M. S. Chae, J. H. Hong, Y. W. Parc, I. S. Ko, S. J. Park, H. J. Qian, W. H. Huang, and C.X. Tang, Emittance growth due to multipole transverse magnetic modes in an rf gun, Phys. Rev. Accel. Beams 14, 104203 (2011).

[44] D. H. Dowell, F. Zhou, and J. Schmerge, Exact cancellation of emittance growth due to coupled transverse dynamics in solenoids and rf couplers, Phys. Rev. Accel. Beams 21, 010101 (2018).

[45] D. H. Dowell, Correcting emittance growth due to stray sextupole fields, arXiv:1810.00086.

[46] D. H. Dowell, Sources of emittance in $\mathrm{rf}$ photocathode injectors: Intrinsic emittance, space charge forces due to non-uniformities, rf and solenoid effects, arXiv:1610.01242.

[47] K. T. McDonald and D. P. Russell, Methods of emittance measurement, in Frontiers of Particle Beams; Observation, Diagnosis and Correction, Lecture Notes in Physics Vol. 343, edited by M. Month and S. Turner (Springer, Berlin, 1989), pp. 122-132.

[48] L. Zheng, J. Shao, Y. Du, J. G. Power, E. E. Wisniewski, W. Liu, C. E. Whiteford, M. Conde, S. Doran, C. Jing, C. Tang, and W. Gai, Experimental demonstration of the correction of coupled-transverse-dynamics aberration in an rf photoinjector, Phys. Rev. Accel. Beams 22, 072805 (2019).

[49] M. Conde, G. Ha, E. Wisniewski, W. Liu, N. Neveu, C. Whiteford, J. Qiu, J. Shao, S. Antipov, L. Zheng et al., Research program and recent results at the Argonne Wakefield Accelerator Facility (AWA), in Proceedings of the 8th International Particle Accelerator Conference (JACoW, Geneva, Switzerland, 2017), WEPAB132.

[50] J. Shao, C. Jing, E. Wisniewski, G. Ha, M. Conde, W. Liu, J. Power, and L. Zheng, Development and high-power testing of an x-band dielectric-loaded power extractor, Phys. Rev. Accel. Beams 23, 011301 (2020).

[51] K. Flöttmann, A space charge tracking algorithm (ASTRA), https://www.desy.de/ mpyflo/.

[52] H. Wiedemann, Particle Accelerator Physics (Springer, New York, 2015), Vol. 3.

[53] C. Gulliford and I. Bazarov, New method for generating linear transfer matrices through combined rf and solenoid fields, Phys. Rev. Accel. Beams 15, 024002 (2012).

[54] PI-MAX/PI-MAX2 System, Princeton Instruments, 2004.

[55] A. Halavanau, G. Qiang, G. Ha, E. Wisniewski, P. Piot, J. G. Power, and W. Gai, Spatial control of photoemitted electron beams using a microlens-array transverseshaping technique, Phys. Rev. Accel. Beams 20, 103404 (2017).

[56] A. Halavanau, Q. Gao, M. Conde, G. Ha, P. Piot, J. G. Power, and E. Wisniewski, Tailoring of an electron-bunch current distribution via space-to-time mapping of a transversely shaped, photoemission-laser pulse, Phys. Rev. Accel. Beams 22, 114401 (2019).

[57] R. Akre, D. Dowell, P. Emma, J. Frisch, S. Gilevich, G. Hays, P. Hering, R. Iverson, C. Limborg-Deprey, H. Loos et al., Commissioning the linac coherent light source injector, Phys. Rev. Accel. Beams 11, 030703 (2008).

[58] H. Trautner, Spectral response of cesium telluride and rubidium telluride photocathodes for the production of highly charged electron bunches, Ph.D. thesis, CERN, Geneva, 2000.

[59] E. E. Wisniewski, D. Velazquez, Z. Yusof, L. Spentzouris, J. Terry, T. J. Sarkar, and K. Harkay, Kelvin probe studies of cesium telluride photocathode for AWA photoinjector, Nucl. Instrum. Methods Phys. Res., Sect. A 711, 60 (2013).

[60] I. Bazarov, L. Cultrera, A. Bartnik, B. Dunham, S. Karkare, Y. Li, X. Liu, J. Maxson, and William Roussel, Thermal emittance measurements of a cesium potassium antimonide photocathode, Appl. Phys. Lett. 98, 224101 (2011).

[61] V. Miltchev, Investigations on the transverse phase space at a photo injector for minimized emittance, Master thesis, Humboldt University, Berlin, 2006.

[62] H. Qian, Research on the emittance issues of photocathode rf gun, Ph.D. thesis, Tsinghua University, 2012.

[63] M. A. H. Schmeißer, In-situ measurements of the intrinsic emittance of photocathodes for high brightness electron beams, arXiv:1809.01390. 